\title{
CONCEPTS
}

\section{Improvising a Posterior Nasal Pack with Equipment in a Basic First Aid Kit}

\author{
Allison K. Royer, MD; Mark C. Royer, MD \\ From the Department of Otolaryngology-Head \& Neck Surgery, Columbus Regional Health, Columbus, IN.
}

\begin{abstract}
Posterior epistaxis is a serious condition that can be difficult to treat in a wilderness setting. The initial standard of care involves packing the affected nostril with a 7 to $9 \mathrm{~cm}$ nasal pack to tamponade the bleed. These packs are often unavailable outside of the emergency or operating room. This study set out to determine whether a posterior nasal pack could be constructed from the supplies present in a basic first aid kit in order to control massive nasal hemorrhage in a wilderness setting. A basic first aid kit was utilized to construct a posterior nasal pack that was inserted into an anatomical model and visibly compared with the Rapid Rhino (Posterior, $7.5 \mathrm{~cm}$; Smith \& Nephew, Austin, TX) nasal packing. The shape, size, and anatomical areas of compression (ie, into nasopharynx and posterior aspect of inferior turbinate) of this pack was similar to the commercially available posterior nasal pack. Placement in an anatomical model appears to provide similar compression as the commercially available posterior pack. This technique may provide short-term hemorrhage control in cases of serious posterior nasal hemorrhage where standard treatment options are not available.
\end{abstract}

Key words: epistaxis, first aid, posterior epistaxis, sphenopalatine artery

\section{Introduction}

Epistaxis is a common condition for which patients seek treatment. Given a variety of factors (eg, altitude, arid climates, etc.), epistaxis is frequently encountered in a wilderness setting. ${ }^{1}$ Epistaxis is classified as either anterior or posterior. Anterior nosebleeds are the most common (90\%), usually occur at the confluence of several superficial vessels near the anterior nasal septum (Kisselbach's plexus), and are easily controlled with anterior pressure, oxymetazoline, and/or anteriorly placed gauze or cotton. ${ }^{1}$

Alternatively, posterior epistaxis is a serious medical emergency. Although posterior epistaxis patients are more likely to be chronically ill (eg, hypertensive, anticoagulated, etc.) than the standard individual enjoying outdoor activities, trauma or failure to take antihypertensives while trekking can result in this type of epistaxis. Posterior epistaxis refers to nasal hemorrhage occurring from branches off the sphenopalatine artery or anterior ethmoid artery (which supply the turbinates, posterior nasal cavity, posterior septum, and nasopharynx) (Figure 1). Etiology of

Corresponding author: Allison K. Royer, MD, Columbus Regional Health, Otolaryngology-Head \& Neck Surgery, 2326 18th St., Columbus, IN 47201 (e-mail: Allison.k.royer@gmail.com).

Submitted for publication March 2016

Accepted for publication June 2016. posterior epistaxis is multifactorial but usually is the result of severe hypertension, anticoagulation, or trauma. Successful treatment requires initial placement of a variety of posterior nasal packs $(7.5-10 \mathrm{~cm}$; eg, Merocel, Epistat, Rapid Rhino, Rhino Rocket) or Foley catheter with petroleum jelly coated strip gauze and may ultimately require interventional radiology embolization or sphenopalatine artery ligation by an otolaryngologist. ${ }^{2}$

Control of anterior packing in a wilderness setting has been well described. ${ }^{1}$ However, treatment of a posterior nasal hemorrhage requires specialized supplies that are usually not brought on routine outings due to space and cost constraints. The purpose of this study was to determine if a posterior nasal pack could be fashioned from basic supplies in an inexpensive first aid kit designed for day hiking.

\section{Methods}

An internet search for "first aid kit hike" on an online shopping website (Amazon.com, Seattle, WA) was performed and the results sorted by price (range: $\$ 7.99$ to \$99.80). The least expensive first aid kit (\$7.99; Lifeline Trail Light Dayhiker First Aid Kit; Wilsonville, OR) was purchased and the contents examined (Figure 2). Select contents of the first aid kit were utilized to fashion a device 


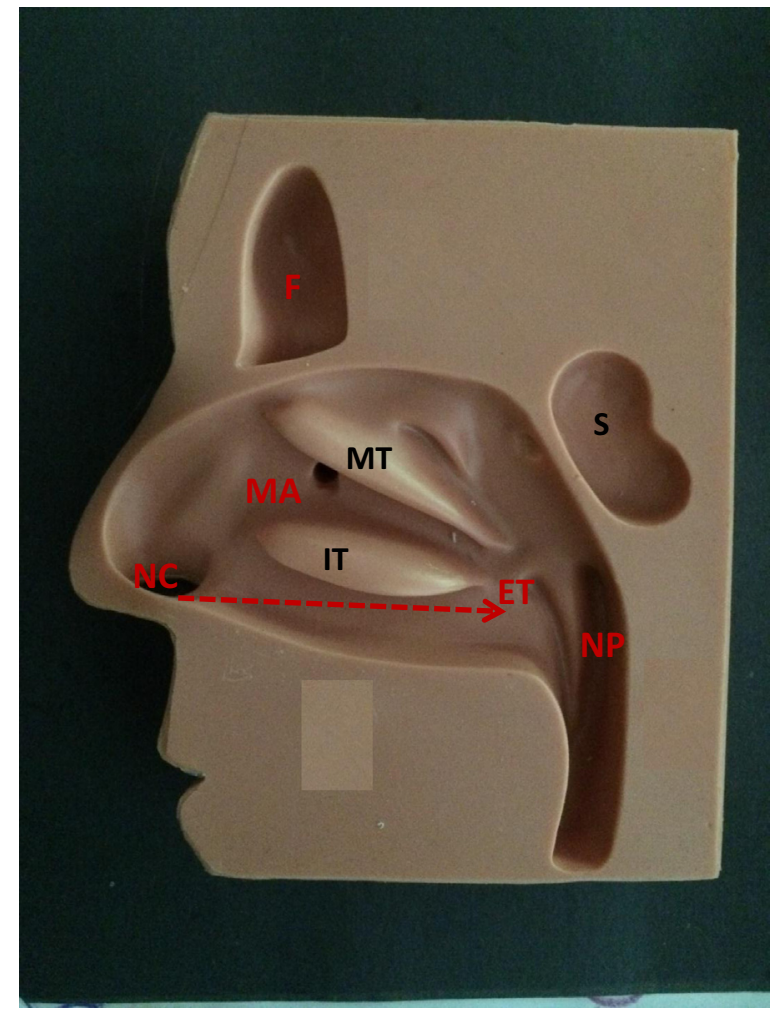

Figure 1. Anatomical model used for nasal packing training (NC, right nasal cavity; F, frontal sinus; $\mathrm{S}$, sphenoid sinus; MT, middle turbinate; IT, inferior turbinate; MA, maxillary antrum; NP, nasopharynx; ET, Eustachian tube opening). Arrow shows direction of packing insertion.

similar to the commercially available nasal pack $(7.5 \mathrm{~cm}$ Rapid Rhino posterior nasal pack, Smith \& Nephew, Austin, TX). This pack was then placed in the anatomical model and compared with the placement of the Rapid Rhino posterior nasal pack.

\section{Results}

A $7.5 \mathrm{~cm}$ nasal pack was successfully fashioned using the supplies in the first aid kit (Figure 3). Three Q-tips, 8 standard adhesive bandages, $30 \mathrm{~cm}$ of $1 / 4$ inch cloth tape, 1 nonadherent pad $(7.6 \mathrm{~cm} \times 10.2 \mathrm{~cm})$, and $20.5 \mathrm{gm}$ packs of triple antibiotic ointment were utilized.

Three Q-tips were secured to each other using cloth tape in order to provide a rigid base for the packing (Figure 3A and B). Eight standard adhesive bandages were then wrapped around this rigid base in order to provide additional rigid support and volume for appropriate compression (Figure 3C). The ends were left slightly tapered, and this was wrapped with a $7.6 \mathrm{~cm} \times 10.5 \mathrm{~cm}$ nonadherent dressing pad (Figure 3D). Additional cloth tape was secured to the proximal end of the packing in order to provide a string for removal (Figure 3F). The nonadherent packing was secured to the core using additional tape at both proximal and distal ends. All of the triple antibiotic ointment in the kit (2 $0.5 \mathrm{~g}$ packs) were opened and used to coat the packing (Figure 3E).

The pack was then inserted in a manner similar to the Rapid Rhino posterior pack. The tip was inserted into the model of the right nares, and gentle but firm pressure was applied to pass the nasal pack parallel to the floor of the nose along the inferior turbinate and septum until it entered the nasopharynx (Figure 4). The ease of insertion, removal, and areas of compression seen on the anatomical model were similar.

\section{Discussion}

Management of posterior epistaxis in a wilderness setting can prove especially challenging. Posterior bleeding requires rapid evacuation to a medical facility as soon as recognized. The equipment necessary to manage this condition is rarely available in a wilderness setting. In this study, we were able to fashion a nasal pack similar in shape and size to a commonly utilized, commercially available posterior nasal pack using the materials in a basic first aid kit. Although the improvised nasal pack is similar in size and appearance to the Rapid Rhino pack, this treatment has not been tested clinically and should only be used in cases of life-threatening hemorrhage when traditional packing is not available. Of note, the improvised pack is untested and theoretically could break apart and result in aspiration if bleeding is severe, especially given the decreased gag reflex present in unconscious individuals.

The Rapid Rhino, nonballoon nasal tampons (eg, Merocel), and Foley catheter with petroleum jelly coated strip gauze methods of treatment for posterior nasal packing have several significant advantages over the improvised pack. First, the Rapid Rhino has an inflation port, which allows additional pressure to be applied to the surrounding nasal mucosa once inserted. Additionally, the Rapid Rhino is coated in carboxymethyl cellulose (a natural hemostatic and lubricating agent). ${ }^{3}$ Occasionally, posterior epistaxis requires placement of a Foley catheter, inflation of the balloon in the nasopharynx, and intranasal packing with petroleumcoated strip gauze around the catheter. This method has advantages of airway protection (balloon in nasopharynx minimizes bleeding into airway) and improved placement of packing around abnormal anatomy (septal deviation/septal spur). Disadvantages include the rare but reported risk of cardiac arrest from vagal stimulation in the nasopharynx. ${ }^{2}$ Nonballoon nasal tampons offer the benefits of increased comfort postinsertion but are 


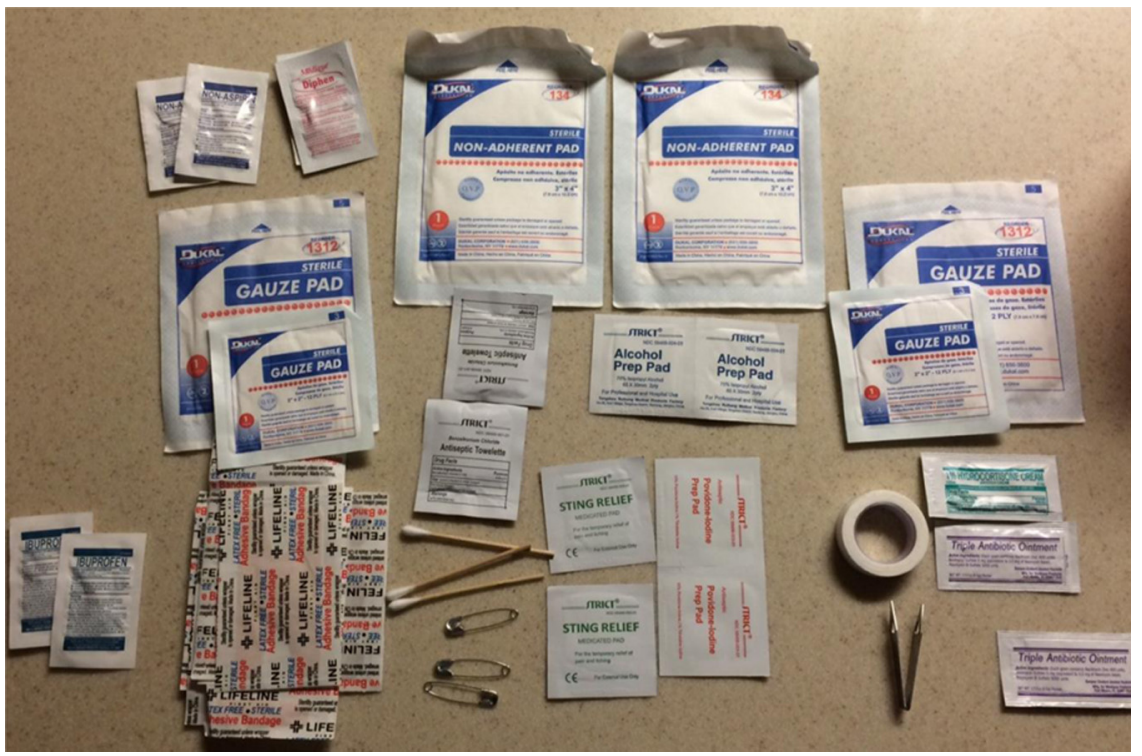

Figure 2. Basic first aid kit contents.

generally less effective at treating severe hemostasis and lack the airway protection of the nasal balloon. ${ }^{3}$

A nonadherent dressing was utilized in this model. However, gauze pads could likewise be substituted and may provide the advantage of improved blood absorption. The number of gauze pads could be varied to increase the size if indicated. Copious placement of triple antibiotic ointment will improve the ease of placement and may provide protection against staphylococcus toxic shock syndrome until antibiotics are able to be administered.
Appropriate placement of such a rigid nasal pack requires caution to avoid pitfalls. First, the pack should be inserted inferiorly and parallel to the floor of the nose (see arrow on Figure 1). Any superior angulation could result in injury to the middle turbinate, ethmoids, and skull base. Any lateral angulation has the potential to enter the orbit (especially in a coexisting naso-orbital ethmoid fracture). Additionally, copious placement of triple antibiotic ointment for lubrication should help prevent further trauma to the mucosa of the inferior turbinate and/or nasal septum.

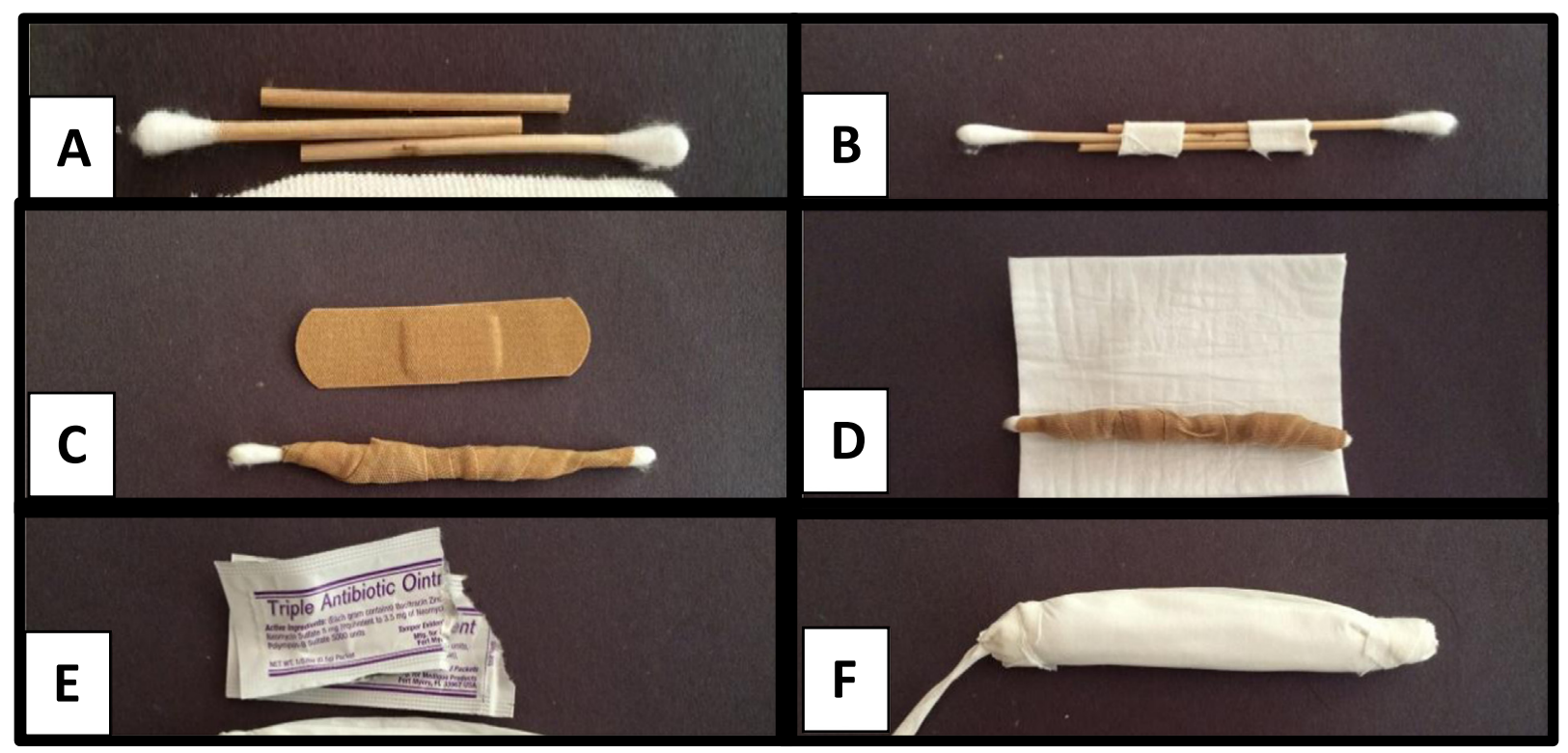

Figure 3. Preparation of posterior nasal pack from first aid supplies. Three Q-tips are fashioned into a $7.5 \mathrm{~cm}$ length rigid support (A,B). Eight standard adhesive bandages are used to secure the Q-tips and increase diameter $(\mathbf{C})$. A nonadherent dressing is wrapped around the rigid support (D) and triple antibiotic added along the length of the packing $(\mathbf{E})$. The completed improvised nasal pack is shown $(\mathbf{F})$. 


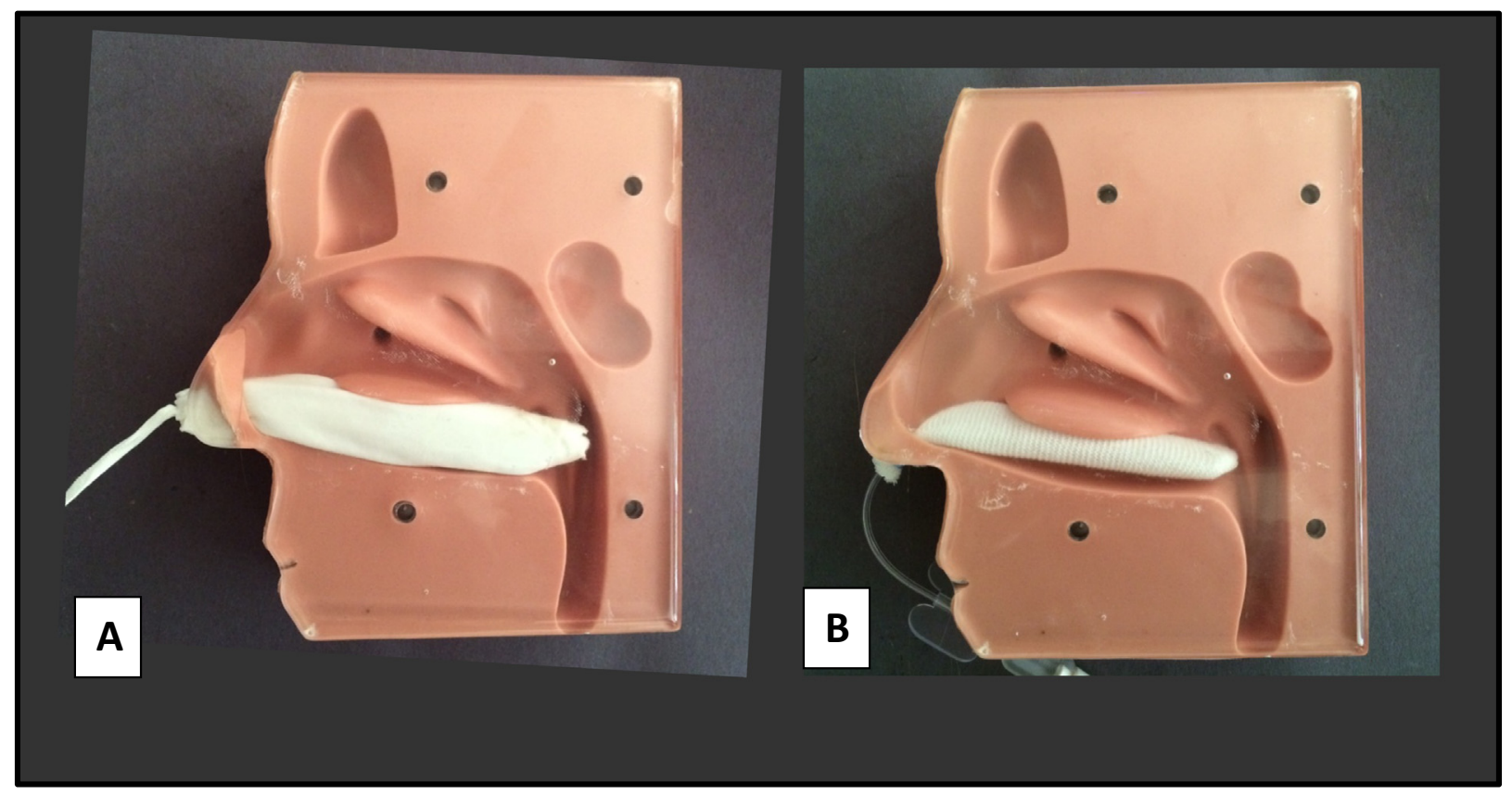

Figure 4. The posterior nasal pack prepared from a basic first aid kit placed in the training model (A) compared with the commercially available posterior nasal pack placed in the same model (B) (not filled with air to demonstrate a pack similar to the Merocel).

Given the pitfalls of posterior nasal pack placement, its use should be reserved for posterior nasal hemorrhage. Anterior (septal) bleeding is easily controlled with anterior pressure. Control of all initial nasal hemorrhage should be attempted by having the patient sit forward and pressure applied to the nasal sidewalls for at least 20 minutes. A posterior bleed is likely if this fails to significantly reduce the bleeding or if there is copious blood noted in the oropharynx.

\section{Conclusion}

Posterior nasal hemorrhage is a life-threatening category of epistaxis. Management requires placement of specialized posterior nasal packs (eg, Rapid Rhino, Merocel, or Foley catheters and petroleum jelly-coated strip gauze) and rapid evacuation to a medical facility. We presented a model for control of posterior epistaxis using the contents of a basic first aid kit. Although this model appears similar in shape, placement method, and anatomical compression to Rapid Rhino posterior nasal pack, further studies are required to ensure patient efficacy and safety. We recommend using this method only if no other standard options are available. To safely manage posterior nasal hemorrhage in a wilderness setting, stocking wilderness medical kits with posterior nasal packs should be considered.

Author Contributions: Study concept and design (AKR; MCR); obtaining funding (N/A); acquisition of the data (MCR); analysis of the data (AKR); drafting of the manuscript (AKR; MCR); critical revision of the manuscript (AKR); and approval of final manuscript (AKR; MCR).

Financial/Material Support: None.

Disclosures: None.

\section{References}

1. Auerbach P, Constance B, Freer L. Field Guide to Wilderness Medicine. 4th ed. St. Louis, MO: Mosby; 2013.

2. Flint PW, ed. Cummings Otolaryngology-Head and Neck Surgery. 5th ed. Philadelphia, PA: Mosby; 2010.

3. Moumoulidis I, Draper MR, Patel H, Jani P, Price T. A prospective randomised controlled trial comparing Merocel and Rapid Rhino nasal tampons in the treatment of epistaxis. Eur Arch Otorhinolaryngol. 2006;263:719-722. 